\title{
Feasibility of dose escalation using intraoperative radiotherapy following resection of large brain metastases compared to postoperative stereotactic radiosurgery
}

\author{
John A. Vargo ${ }^{1}$, Kristie M. Sparks ${ }^{1}$, Rahul Singh ${ }^{2}$, Geraldine M. Jacobson ${ }^{1}$, Joshua D. \\ Hack $^{1}$, and Christopher P. Cifarelli ${ }^{2}$ \\ ${ }^{1}$ Department of Radiation Oncology, West Virginia University, 1 Medical Center Dr., Morgantown, \\ WV 26505, USA \\ ${ }^{2}$ Department of Neurosurgery, West Virginia University, Morgantown, WV, USA
}

\section{Abstract}

Background and purpose-Post-operative SRS (stereotactic radiosurgery) for large brain metastases is challenged by risks of radiation necrosis that limit SRS dose. Intraoperative radiotherapy (IORT) is a potential alternative, however standard dose recommendations are lacking.

Methods and materials-Twenty consecutive brain metastases treated with post-operative SRS were retrospectively compared to IORT plans generated for 10-30 Gy in 1 fraction to $0-5 \mathrm{~mm}$ by estimating the applicator size and distance from critical organs using pre-operative and postoperative MRI. Additionally, 7 consecutive patients treated with IORT 30 Gy to surface were compared to retrospectively generated SRS plans using the post-operative MRI to 15-20 Gy and 30 Gy in 1 fraction marginal dose.

Results-For the 20 resection cavities treated with SRS and retrospectively compared to IORT, IORT from 10 to $30 \mathrm{~Gy}$ resulted in lower or not significantly different doses to the optic apparatus and brainstem. Comparatively for the 7 patients treated with IORT 30 Gy to retrospective SRS plans to standard 15-20 Gy and 30 Gy marginal dose, IORT resulted in significantly lower doses to the optic apparatus and brainstem. At a median follow-up of 6.2 months, $86 \%$ of patients treated with surgery and IORT achieved local control and $0 \%$ developed radiographic or symptomatic radiation necrosis.

Conclusions-Critical organ dosimetry for IORT remains generally lower than that achieved with single fraction SRS following resection of large brain metastases. We recommend $30 \mathrm{~Gy}$ to surface as the preferred prescription, consistent with the dose recommendation for IORT in

\footnotetext{
Conflict of interest John A Vargo, MD receives speaking honoraria from BrainLAB. All remaining authors declare they have no conflicts of interest.

Compliance with ethical standards

Ethical approval All procedures performed in studies involving human participants were in accordance with the ethical standards of the institutional and/or national research committee and with the 1964 Helsinki declaration and its later amendments or comparable ethical standards.

Informed consent Informed consent was obtained from all individual participants included in the study for treatment delivery.
} 
glioblastoma used in the ongoing INTRAGO-II phase-III trial. Early clinical outcomes appear promising for surgery and IORT.

\section{Keywords}

Brain metastases; Stereotactic radiosurgery; GammaKnife; Intraoperative radiation; Resection cavity

\section{Introduction}

Multiple landmark clinical trials have established the importance of aggressive local therapy via surgery and/or stereotactic radiosurgery (SRS) with the omission of whole brain radiotherapy in sparing long-term neurocognitive effects for patients with brain metastases [1-3]. Recent prospective data from RTOG 1270/NCCTG N107C comparing SRS to the resection cavity versus whole brain radiotherapy, again highlighted the importance of SRS in preserving neurologic function [4]. However, the preservation of neurocognition without a compromise in overall survival came at a cost of worse local control. Due to risks of radiation necrosis, SRS dose especially for large resection cavities is constrained to 12-20 Gy in 1 fraction with lower doses used with increasing resection cavity sizes [4]. However, SRS remains a superior alternative to observation following the resection of brain metastases, as $50-60 \%$ of patients observed after surgery alone will suffer local recurrence [5]. Both trials reduced the SRS dose with increasing cavity size to reduce the risk of necrosis, though this is contrary to the principle that greater tumor volume should require a higher dose for local control. This dose reduction combined with difficulties in target delineation of the resection cavity and challenges of tumor cell hypoxia in the post-operative setting may have combined to account for the up to $20-40 \%$ rates of local failure [4, 5]. Thus, alternative strategies are warranted.

Intraoperative radiotherapy (IORT) has been used as a potential alterative to SRS following resection of large brain tumors [6-12]. IORT has advantages of eliminating challenges in target definition, steep conformal dose delivery that may afford dose-escalation relative to SRS, and increased patient convenience by integrating resection and radiotherapy into 1 procedure. A variety of techniques have been used for IORT in brain metastases including low-energy photons and permanent low dose rate brachytherapy with ${ }^{131} \mathrm{Cs}$ and ${ }^{125} \mathrm{I}$ [6-8, $11,12]$. However, dose selection for low energy photon based IORT in brain tumors remains largely empiric with doses ranging from 10 to $30 \mathrm{~Gy}$ in 1 fraction to varying prescription depths of $0-5 \mathrm{~mm}$ [6-10]. Thus to help better guide the clinical application of IORT following resection of large brain metastases, we aimed to compare the critical organ dosimetry for varying prescription doses of IORT to patients treated with single fraction SRS. We hypothesize that the combination of steep conformal dose fall-off and the compression of the resection cavity inherent to IORT may allow for dose escalation beyond the standard dose range of 12-20 Gy used for post-operative SRS with less dose to surrounding critical organs. 


\section{Materials and methods}

Following institutional review board approval, twenty consecutive brain metastases resection cavities treated with Leksel GammaKnife ${ }^{\circledR}$ Perfexion ${ }^{\text {TM }}$ (Elekta, Stockholm Sweden) SRS from 2013 to 2017 were retrospectively reviewed. Patients with significant residual disease greater than $2 \mathrm{~mm}$ in thickness that would have precluded IORT were excluded. There was no exclusion by size, location, or number of metastases. SRS doses ranged from 12 to $22 \mathrm{~Gy}$ in 1 fraction to the 50\% isodose line. For SRS, the gross tumor volume (GTV) was defined as the resection cavity plus any minimal residual tumor; GTV equaled the planning target volume with no additional expansion routinely applied. Critical organ dosimetry was retrospectively collected and compared to theoretical IORT plans generated using $50 \mathrm{kV} \mathrm{X-}$ rays on the INTRABEAM ${ }^{\circledR} 600$ (ZEISS International, Jena, Germany). IORT plans were generated using spherical applicators ranging from 1.5 to $5 \mathrm{~cm}$ for doses ranging from 10-30 Gy in 1 fraction prescribed to $0-5 \mathrm{~mm}$ from the applicator surface by estimating the applicator size and distance from critical organs using a combination of pre-operative and post-operative MR imaging. Figure 1 shows a case example of IORT for brain metastasis.

Additionally, 7 consecutive patients treated with surgical resection followed by IORT using Zeiss INTRA-BEAM ${ }^{\circledR}$ spherical applicator system to deliver $30 \mathrm{~Gy}$ to applicator surface for large brain metastases from October 2017 to April 2018 were retrospectively reviewed. Using the post-operative MRI, single fraction SRS plans were created for the GammaKnife ${ }^{\circledR}$ Perfexion using GammaPlan v10.1 (Elekta, Stockholm, Sweden). SRS plans were generated both for marginal doses of 15-20 Gy in 1 fraction prescribed to the 50\% isodose line as dictated by the resection cavity volume per NCCTG N107C dose guidelines as well for $30 \mathrm{~Gy}$ marginal dose as used for IORT [4]. Critical organ dosimetry for IORT was calculated using intraoperative measurements of the minimum distance to the respective critical organ to the resection cavity using real time intraoperative neuro-navigation (BrainLAB® Cranial Navigation, Munich Germany) (see Fig. 1).

Statistical analyses were completed using IBM SPSS version 24 (SPSS Chicago, Illinois). Mean maximal doses to optic chiasm, optic nerve, and brainstem as well as the volume of brain receiving $12 \mathrm{~Gy}$ (V12Gy) were compared between SRS and IORT using two-sided paired $t$ tests. For patients with multiple metastases, including those with multiple resection cavities, critical organ dosimetry was calculated for each resection cavity only without contribution from other lesions. For SRS, the brain total V12Gy was the total intracranial volume receiving $12 \mathrm{~Gy}$ as estimated from the skull contour [13]. For comparison, an alternative definition of V12 (SRS V12Gy Minus GTV) was calculated using total V12Gy volume minus the GTV volume [14]. For IORT, the V12Gy was conservatively calculated using a spherical estimate of the V12Gy excluding the applicator volume, and thus does not account for attenuation at high density interfaces such as the skull. A $p<0.05$ was considered significant for all analyses.

\section{Results}

Baseline patient characteristics for the 19 patients (20 resection cavities) treated with SRS and retrospectively compared to IORT were as follows: median patient age was 60 [inter- 
quartile range (IQR): 49-62], RPA class 2 (IQR 2-2), and GPA class 2.0 (IQR 1-2). The most common histology was non-small cell lung cancer. The median pre-operative maximal tumor diameter was $3.4 \mathrm{~cm}$ (IQR 2.5-4.1), and median post-operative maximal resection cavity diameter was $2.4 \mathrm{~cm}$ (IQR 1.6-2.7). The estimated IORT applicator median was 2.5 (IQR 2.5-3.9). The median planning target volume at the time of SRS was $7.2 \mathrm{cc}$ (IQR 4.29.2). The median marginal dose for SRS was $17.0 \mathrm{~Gy}$ (IQR 15.0-19.5) to the $50 \%$ isodose line, with a corresponding median maximum dose of $34.0 \mathrm{~Gy}$ (IQR 30.0-39.0 Gy). The mean maximal dose to optic chiasm, optic nerve, brainstem, and V12Gy were 0.50 Gy (IQR 0.23-0.68), 0.41 Gy (IQR 0.21-0.53), 2.44 Gy (IQR 0.34-2.56), and 19.74 cc (IQR 10.5524.67) respectively.

As summarized in Table 1, IORT across the dose range of 10-30 Gy resulted in lower or not statistically significantly different mean maximal doses to optic chiasm, optic nerve, and brainstem. The mean total V12Gy was lower or not statistically significantly different comparing IORT from 10 to $20 \mathrm{~Gy}$ (see Table 1). For IORT doses of $30 \mathrm{~Gy}$ to the applicator surface, the mean total V12Gy was statistically significantly higher than SRS (28.94 cc \pm 21.78 versus $19.74 \mathrm{cc} \pm 15.35, p=0.02$ ). When splitting data by applicator size $\geq 4 \mathrm{~cm}$, no significant differences in mean total V12Gy were noted comparing IORT 30 Gy to the applicator surface versus SRS for applicators $<4 \mathrm{~cm}(18.91 \mathrm{cc} \pm 9.76$ versus $16.41 \mathrm{cc}$ $\pm 9.04, p=0.36$ ); while for those $\geq 4 \mathrm{~cm}$ the mean total V12Gy remained significantly higher for IORT $30 \mathrm{~Gy}$ to the applicator surface versus SRS (59.03 $\mathrm{cc} \pm 20.78$ versus 29.74 $\mathrm{cc} \pm 25.83, p<0.01$ ). When using the alternative SRS definition of V12Gy Minus GTV, significantly higher doses of V12Gy were noted for IORT prescriptions of 30 Gy to surface, $16 \mathrm{~Gy}$ to $2 \mathrm{~mm}$, and $18 \mathrm{~Gy}$ to $2 \mathrm{~mm}$ compared to SRS; with no significant differences for IORT prescriptions of $20 \mathrm{~Gy}$ to surface, $10 \mathrm{~Gy}$ to $5 \mathrm{~mm}$, and $14 \mathrm{~Gy}$ to $2 \mathrm{~mm}$ (see Table 1). When splitting data by applicator size $\geq 4 \mathrm{~cm}$ with SRS definition of V12Gy Minus GTV, IORT prescriptions of $16 \mathrm{~Gy}$ to $2 \mathrm{~mm}$ and $18 \mathrm{~Gy}$ to $2 \mathrm{~mm}$ were not significantly different than SRS for applicators $<4 \mathrm{~cm}(10.45 \mathrm{cc} \pm 5.09$ versus $10.18 \pm 5.39, p=0.85)$ and $(13.46$ $\mathrm{cc} \pm 6.54$ versus $10.18 \pm 5.39, p=0.06$ ) respectively; while IORT prescription of 30 Gy to surface was statistically higher even for applicators $<4 \mathrm{~cm}$ (18.91 $\mathrm{cc} \pm 9.76$ versus 10.18 $\pm 5.39, p<0.01)$. For applicators $\geq 4 \mathrm{~cm}$ and using the V12Gy Minus GTV definition for SRS, IORT to 16 Gy to $2 \mathrm{~mm}, 18 \mathrm{~Gy}$ to $2 \mathrm{~mm}$, and $30 \mathrm{~Gy}$ to surface was consistently statistically significantly higher than SRS (30.63 cc \pm 7.74 versus $15.71 \pm 8.73, p<0.01)$, (39.50 $\mathrm{cc} \pm 10.24$ versus $15.71 \pm 8.73, p<0.01)$, and (59.03 $\mathrm{cc} \pm 20.28$ versus $15.71 \pm 8.73$, $p<0.01$ ), respectively.

For the 7 patients treated with IORT 30 Gy to surface for large brain metastases, the median patient age was 67 (IQR 44-75), median RPA class was 2 (IQR 2-2), and median SRS gross tumor volume was $15.58 \mathrm{cc}$ (IQR 6.18-19.36). The most common primary histology was non-small cell lung cancer in $71 \%$. Applicator size ranged from 1.5 to $3.0 \mathrm{~cm}$. As highlighted in Fig. 2, the mean V12Gy volume for IORT with SRS definition of V12 total was $18.79 \mathrm{cc} \pm 10.37$ versus $25.78 \mathrm{cc} \pm 10.51$ for SRS marginal doses of $15-20 \mathrm{~Gy}, p=$ 0.09 , and $65.24 \mathrm{cc} \pm 33.58$ for SRS doses of $30 \mathrm{~Gy}, p<0.01$. The mean V12Gy volume for IORT with SRS definition of V12 Minus GTV was $18.79 \mathrm{cc} \pm 10.37$ versus $12.84 \mathrm{cc} \pm 3.78$ for SRS marginal doses of 15-20 Gy, $p=0.14$, and $52.29 \mathrm{cc} \pm 26.68$ for SRS doses of $30 \mathrm{~Gy}, p<$ 0.01 . The mean maximal dose for the brainstem for IORT was $0.34 \mathrm{~Gy} \pm 0.46$ versus 0.97 
\pm 0.75 for SRS marginal doses of $15-20 \mathrm{~Gy}, p<0.01$, and $1.82 \mathrm{~Gy} \pm 1.56$ for SRS doses of $30 \mathrm{~Gy}, p=0.01$. The mean maximal dose for the optic apparatus for IORT was $0.26 \mathrm{~Gy}$ \pm 0.25 versus $0.60 \pm 0.38$ for SRS marginal doses of $15-20 \mathrm{~Gy}, p=0.04$, and $1.13 \mathrm{~Gy} \pm 0.71$ for SRS doses of $30 \mathrm{~Gy}, p<0.01$.

At a median follow-up of 6.2 months (IQR 3.6-8.8), 86\% of the patients treated with IORT remain alive living and well and $0 \%$ have developed radiographic or symptomatic radiation necrosis from IORT. Figure 3 shows an example of IORT noting the potential clinical advantages in terms of radiation injury reactions and associated bleeding of adjacent hemorrhagic metastases which developed symptomatic hemorrhage despite SRS and whole brain irradiation. The 1 patient who expired, died from cardiopulmonary arrest. One patient developed local failure after surgery and IORT at 8.8 months following surgery and IORT for a dural-based metastasis secondary to endometrial cancer (see Fig. 4); thus the crude local control rate was $86 \%$.

\section{Discussion}

The recently published RTOG 1270/NCCTG N107C highlighted the importance of postoperative SRS following surgical resection of brain metastases in preservation of neurocognitive quality of life over post-operative whole brain irradiation, at the expense of suboptimal local control [4]. IORT represents a potential alternative to SRS with a steep conformal dose fall-off inherent to the low-energy $50 \mathrm{kV}$ X-ray source. Clinically, the compression of the resection cavity maximizes dose to the target while obviating challenges in SRS target definition, and increases patient convenience over SRS. Furthermore, from a radiobiological perspective, immediate IORT may potentially improve local control by counteracting the tumor cell proliferation caused by the surgical manipulation of the microenvironment [15]. However, the doses used in the IORT brain tumor literature have varied significantly from 10 to $30 \mathrm{~Gy}$ prescribed to $0-5 \mathrm{~mm}$ from the applicator surface [610]. Here, we retrospectively compared the dose to critical organs and V12Gy for IORT versus that which was achieved with single fraction SRS. As summarized in Table 1, IORT across the dose ranges reported in the literature resulted in lower or not statistically significantly different doses to critical organs than that achieved with SRS; with optic chiasm doses generally $1 / 2$, optic nerve $1 / 2$ to $1 / 3 \mathrm{rd}$, and brainstem $1 / 2$ to the same as the SRS dose. This retrospective comparison was then validated in a preliminary cohort of 7 consecutive patients treated with IORT $30 \mathrm{~Gy}$ to the applicator surface compared to retrospective created SRS plans to margins doses of 15-20 Gy and $30 \mathrm{~Gy}$ (see Fig. 2); again with $1 / 2$ to $1 / 3$ rd the dose to optic apparatus and brainstem with IORT compared to SRS 1520 Gy marginal dose and a significant reduction of $1 / 4$ to $1 / 6$ th the dose compared to SRS 30 Gy marginal dose. V12Gy doses were generally comparable from clinical SRS to IORT 10-30 Gy to $0-5 \mathrm{~mm}$ depth except for the largest applicators $\geq 4 \mathrm{~cm}$, comparable for IORT to 30 Gy clinical cohort to SRS marginal doses of 15-20 Gy, and significantly lower for IORT to $30 \mathrm{~Gy}$ clinical cohort to SRS marginal doses of $30 \mathrm{~Gy}$. Clinically, the early outcomes following surgery and IORT appear to promisingly validate these dosimetric comparisons with $86 \%$ local control and $0 \%$ radiographic or symptomatic radiation necrosis. 
IORT has been used for decades in the treatment of brain metastases, a needle applicator mounted to a stereotactic frame was placed into the center of the tumor prior to resection, using doses ranging from 10 to $20 \mathrm{~Gy}$ to $2 \mathrm{~mm}$ depth, local control rates ranged from 81 to $100 \%$ for metastases with radiation necrosis rates of $<5 \%[7,16,17]$. Promising results for the use of more recently developed spherical applicator systems better able to conform to the resection cavity than needle applicators from the TARGIT-A randomized trial using 20 Gy to surface in early-stage breast cancer has brought renewed interest to the use of IORT [18]. Investigators from Cleveland Clinic recently published a phase I feasibility trial using the INTRABEAM delivery system and the associated spherical applicator system for resected brain metastases [6]. Using a standardized dose of $14 \mathrm{~Gy}$ to $2 \mathrm{~mm}$ "chosen on the basis of previous experience reported in the literature and on experience with SRS," IORT resulted in a $30 \%$ crude local failure rate at a mean of 9 months post-IORT. Conversely, high rates of radiation necrosis were reported in a phase I dose-escalation study for recurrent (often previously-irradiated) pediatric brain tumors, where $10 \mathrm{~Gy}$ prescribed to $5 \mathrm{~mm}$ depth resulted in a $21 \%$ rate of radiation necrosis [9]. An ongoing international phase III clinical trial (INTRAGO-II, NCT02685605) is investigating the potential role of IORT for glioblastoma, where $30 \mathrm{~Gy}$ to the applicator surface is prescribed prior to $60 \mathrm{~Gy}$ of conventionally fractionated external beam radiotherapy. The dose of $30 \mathrm{~Gy}$ to surface was selected based on the preceding phase I/II dose-escalation study from the same group [10]. The results presented here-in would support that $30 \mathrm{~Gy}$ to the applicator surface provides a dose to critical organs lower than or comparable to that achieved with SRS to standard marginal doses, and except for large applicator diameters, $\geq 4 \mathrm{~cm}$, achieves a comparable V12Gy. Clinically the use of 30 Gy to surface IORT resulted in comparably lower rates of local failure at $14 \%$ with 6 -months follow-up and $0 \%$ radiation necrosis. With the suboptimal local control noted with $14 \mathrm{~Gy}$ to $2 \mathrm{~mm}$, the higher rates of radiation necrosis noted with $10 \mathrm{~Gy}$ to $5 \mathrm{~mm}$, and the established safety of $30 \mathrm{~Gy}$ to surface from the glioblastoma literature, we recommend the use of $30 \mathrm{~Gy}$ to surface when applying IORT for brain metastases recognizing that this will achieve comparable if not lower critical organ dosimetry to standard marginal doses for SRS. For larger tumors requiring applicators $\geq 4$ $\mathrm{cm}$, we recommend decreasing the dose to $20 \mathrm{~Gy}$ to surface to conservatively maintain a volume of normal brain receiving $12 \mathrm{~Gy}$ that is comparable to that achieved clinically with SRS.

A surface based prescription for IORT is supported by multiple pathology series which document that an infiltration depth of $2 \mathrm{~mm}$ is described as deep [19-21]. Considering that IORT prescribed to $30 \mathrm{~Gy}$ at the applicator surface would provide an estimated 19.9-23.5 Gy at $2 \mathrm{~mm}$ depth; these pathology data would support that any unresected microscopic tumor infiltration would receive sufficient toxic tumor dose. Eventually as highlighted by the 1 recurrence noted herein for a dural-based endometrial cancer metastasis, ideally IORT prescriptions could be modulated as specified by tumor pathology recognizing the heterogeneity in growth and infiltration by various tumor subtypes and the potential impact on local failure [22].

A number of alternative techniques to low energy X-ray based IORT have been described for the treatment of brain metastases including permeant low dose rate brachytherapy with ${ }^{125} \mathrm{I}$ and ${ }^{131} \mathrm{Cs}$ as well as temporary implants with balloon based delivery of aqueous ${ }^{125} \mathrm{I}$ [6-8, 
$11,12]$. Low energy X-ray based IORT has advantages of avoiding concerns of seed migration, no need for special handling precautions of radioactive sources, and eliminates the complexity of seed placement thereby reducing anesthesia time and potential reoperation for seed removal in temporary brachytherapy implants. Potential disadvantages compared to brachytherapy forms of IORT include the spherical applicator system is not able to be customized to conform to irregularly shaped cavities and theoretical concerns for trauma to normal brain tissue with spherical applicator placement or if shifts were to occur during placement.

This study is limited by retrospective design. Applicator diameter was estimated based on a combination of preoperative and post-operative MRI and thus may not account for true applicator size with the intraoperative compression often clinically afforded by IORT.

Reliable dosimetric correlates to predict risks of radiation necrosis for IORT are currently lacking, thus it is possible that the V12Gy may not correlate with rates of radiation necrosis following IORT. Furthermore, it is difficult to directly compare the V12Gy for SRS versus IORT. For IORT, the high dose region beyond the applicator surface that interfaces with the resection cavity thickness could not be reliably measured and thus any cavity thickness or residual tumor was not excluded from the calculated V12Gy for IORT; nor was the dose extending into air beyond the applicator entrance or attenuated at the skull interface. Nonetheless, using either V12Gy calculated as a total volume or V12Gy Minus GTV for SRS results were generally consistent; IORT V12Gy was comparable or lower except for the highest dose range with applicators $\geq 4 \mathrm{~cm}$. SRS target definition here-in did not include a 2 $\mathrm{mm}$ margin as recommended by some when target resection cavities and thus the doses to critical organs and V12Gy may have been lower here than in other SRS experiences [19, 23]. However, even in prospective clinical trials the margin for post-operative SRS for brain metastases remains variable $[4,5]$. Other critical organs were not compared in this study such as the scalp, ocular structures (such as lens or retina), or cochlea. Due to the low penetration through high density material such as the skull of the $50 \mathrm{kV}$ X-rays used in IORT, scalp, ocular structures, or cochlea dose is always assumed to be lower than SRS [20, 24]. Furthermore, the relative biologic effectiveness of IORT versus SRS beam profiles were not accounted for, wherein at distances greater than 1-1.5 cm (such as was the case for most of the critical organs studied) the decreased relative biologic effectiveness of IORT $50 \mathrm{kV} \mathrm{X-}$ rays may further reduced the effective dose to critical organs and volume of normal brain receiving 12 Gy relative to that measured here-in [21, 25]. Continued study is needed to best define the optimal application and dose selection for low energy X-ray IORT in the treatment of brain metastases.

\section{Conclusions}

IORT prescribed $30 \mathrm{~Gy}$ to the applicator surface provides lower doses to the optic apparatus and brainstem than that clinically achieved with SRS. The V12Gy is a function of applicator size, and when applicator size exceeds $\geq 4 \mathrm{~cm}, 30 \mathrm{~Gy}$ to surface results in mean doses of V12Gy higher than that clinically achieved with SRS. Consistent with the dose recommendation for IORT in glioblastoma used in the ongoing INTRAGO-II prospective randomized trial, we recommend $30 \mathrm{~Gy}$ to surface as the preferred prescription dose for 
IORT in brain metastases as supported by the narrow range of infiltration reported in pathologic series and the promising early clinical outcomes reported herein.

\section{References}

1. Kocher M, Soffietti R, Abacioglu U et al. (2011) Adjuvant whole brain radiotherapy versus observation after radiosurgery or surgical resection of one to three cerebral metastases: results of the EORTC 22952-26001 study. J Clin Oncol 29:134-141 [PubMed: 21041710]

2. Brown PD, Jaeckle K, Ballman KV et al. (2016) Effect of radiosurgery alone vs radiosurgery with whole brain radiation therapy on cognitive function in patients with 1 to 3 brain metastases: a randomized clinical trial. JAMA 316:401-409 [PubMed: 27458945]

3. Chang EL, Wefel JS, Hess KR et al. (2009) Neurocognition in patients with brain metastases treated with radiosurgery or radiosurgery plus whole-brain irradiation: a randomized contolled trial. Lancet 10:1037-1044 [PubMed: 19801201]

4. Brown PD, Ballman KV, Cerhan J et al. (2017) N107C/CEC.3: a Phase III trial of post-operative stereotactic radiosurgery (SRS) compared with whole brain radiotherapy (WBRT) for resected metastatic brain disease. Lancet Oncol 18:1049-1060 [PubMed: 28687377]

5. Mahajan A, Ahmed S, McAleer MF et al. (2017) Post-operative stereotactic radiosurgery versus observation for completely resected brain metastases: a single-centre, randomised, controlled, phase 3 trial. Lancet Oncol 18:1040-1048 [PubMed: 28687375]

6. Weil RJ, Mavinkurve CG, Chao ST et al. (2015) Intraoperative radiotherapy to treat newly diagnosed solitary brain metastasis: initial experience and long-term outcomes. J Neurosurg 122:825-832 [PubMed: 25614945]

7. Curry WT, Jr, Cosgrove GR, Hochberg FH et al. (2005) Stereotactic interstitial radiosurgery for cerebral metastases. J Neurosurg 103:6305

8. Pantazis G, Trippel M, Birg W et al. (2009) Stereotactic interstitial radiosurgery with the photon radiosurgery system (PRS) for metastatic brain tumors: a prospective single-center clinical trial. Int J Radiat Oncol Biol Phys 75:1392-1400 [PubMed: 19464825]

9. Kalapurakal JA, Goldman S, Stellpfung W et al. (2006) Phase I study of intraoperative radiotherapy with photon radiosurgery system in children with recurrent brain tumors: preliminary report of first dose level (10 Gy). Int J Radiat Oncol Biol Phys 65:800-808 [PubMed: 16580791]

10. Giordano FA, Brehmer S, Abo-Madyan Y et al. (2014) INTRAGO: intraoperative radiotherapy in glioblastoma multiforme: a phase I/II dose escalation study. BMC Cancer 14:992 [PubMed: 25535398]

11. Wernicke AG, Hirschfeld CB, Smith AW et al. (2017) Clinical outcomes of large brain metastases treated with neurosurgical resection and intra-operative Cesium-131 brachytherapy: results of a prospective trial. Int J Radiat Oncol Biol Phys 98:1059-1068 [PubMed: 28721889]

12. Raleigh DR, Seymore ZA, Tomlin B et al. (2017) Resection and brain brachytherapy with permanent idodine-125 sources for brain metastasis. J Neurosurg 126:1749-1755 [PubMed: 27367240]

13. Flickinger JC, Kondziolka D, Maitz AH, Lunsford LD (1998) Analysis of neurological sequelae from radiosurgery of arteriovenous malformations: how location affects outcome. Int J Radiat Oncol Biol Phys 40:273-278 [PubMed: 9457809]

14. Levegrun S, Hof H, Essig M, Sclegel W, Debus J (2004) Radiation-induced changes of brain tissue after radiosurgery in patients with arteriovenous malformations: correlation with dose distribution parameters. Int J Radiat Oncol Biol Phys 59:796-808 [PubMed: 15183483]

15. Belletti B, Vaidya JS, D'Andrea S et al. (2008) Targeted intraoperative radiotherapy impairs the stimulation of breast cancer cell proliferation and invasion caused by surgical wounding. Clin Cancer Res 14:1325-1332 [PubMed: 18316551]

16. Di Lorenzo N, Cavedon C, Paier F et al. (2004) Interstitial radiosurgery with the photon radiosurgery system in the minimally invasive treatment of selected deep-seated brain tumors. $\mathrm{J}$ Chemother 16:70-74 
17. Gallina P, Franceson P, Cavedon C et al. (2002) Stereotactic interstitial radiosurgery with a miniature X-ray device in the minimally invasive treatment of selected tumors in the thalamus and the basal Ganglia. Stereotact Funt Neurosurg 79:202-213

18. Vaidya JS, Wenz F, Bulsara M et al. (2014) Risk-adapted targeted intraoperative radiotherapy versus whole-breast radiotherapy for breast cancer: 5-year results for local control and overall survival from the TARGIT-A randomised trial. Lancet 383:603-613 [PubMed: 24224997]

19. Siam L, Bleckmann A, Chaung HN et al. (2015) The metastatic infiltration at the metastasis/brain parenchyma-interface is very heterogeneous and has a significant impact on survival in a prospective study. Oncotarget 6:29254-29267 [PubMed: 26299612]

20. Raore B, Schniederjan M, Prabhu R et al. (2011) Metastasis infiltration: an investigation of the postoperative brain-tumor interface. Int J Radiat Oncol Biol Phys 81:1075-1080 [PubMed: 20971574]

21. Baumert BG, Rutten I, Dehing-Oberije C et al. (2006) A pathology-based substrate for target definition in radiosurgery of brain metastases. Int J Radiat Oncol Biol Phys 66:187-194 [PubMed: 16814946]

22. Brennan C, Yang TJ, Hilden P et al. (2014) A phase 2 trial of stereotactic radiosurgery boost after surgical resection for brain metastases. Int J Radiat Oncol Biol Phys 88:130-136 [PubMed: 24331659]

23. Choi CY, Chang SD, Gibbs IC et al. (2012) Stereotactic radiosurgery of the postoperative resection cavity for brain metastases: prospective evaluation of target margin on tumor control. Int J Radiat Oncol Biol Phys 84:336-342 [PubMed: 22652105]

24. Chen Y, Souri S, Dian X et al. (2016) SU-F-T-56: Dosimetric characterization of the INTRABEAM $50 \mathrm{kV}$ Xray system with a needle applicator in heterogeneous tissues. Med Phys 43:3474

25. Liu Q, Schneider F, Ma L, Wenz F, Herskind C (2013) Relative biologic effectiveness (RBE) of 50 $\mathrm{kV}$ X-rays measured in a phantom for intraoperative tumor-bed irradiation. Int J Radiat Oncol Biol Phys 85:1127-1133 [PubMed: 22981707] 

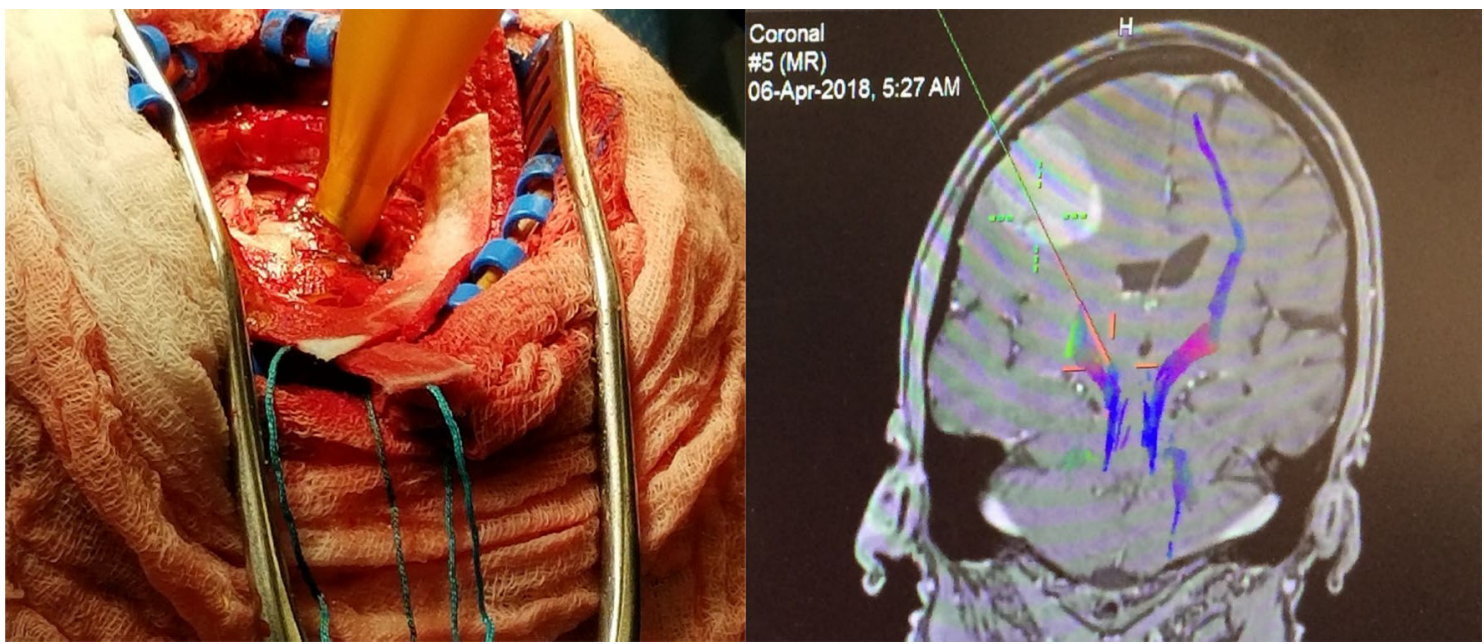

Fig. 1.

Case example of the spherical applicator used for IORT for brain tumor patient 
A Comparison of Brain V12 with SRS Total V12 (cc)

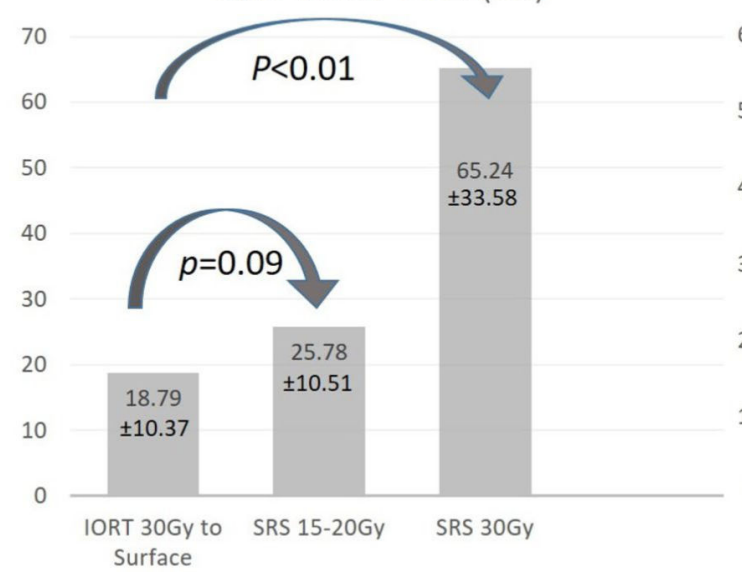

C Comparison of Maximal Dose to Brainstem (Gy)

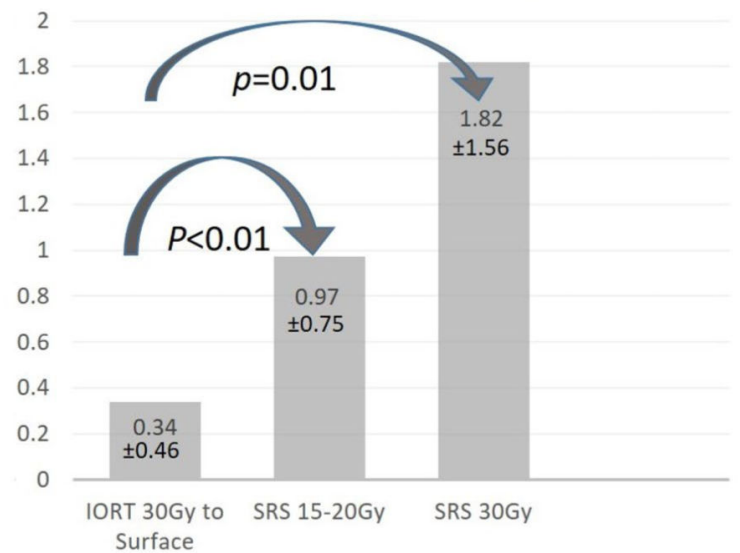

B Comparison of Brain V12 with SRS V12Gy sub GTV (cc)

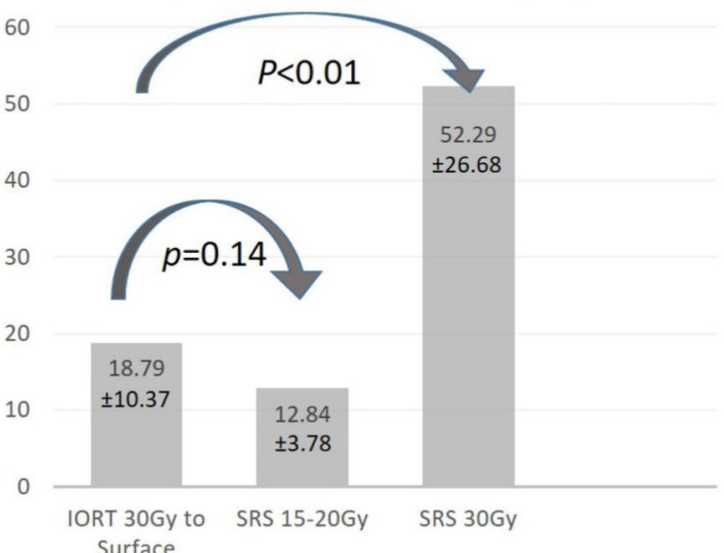

D Comparison of Maximal Dose

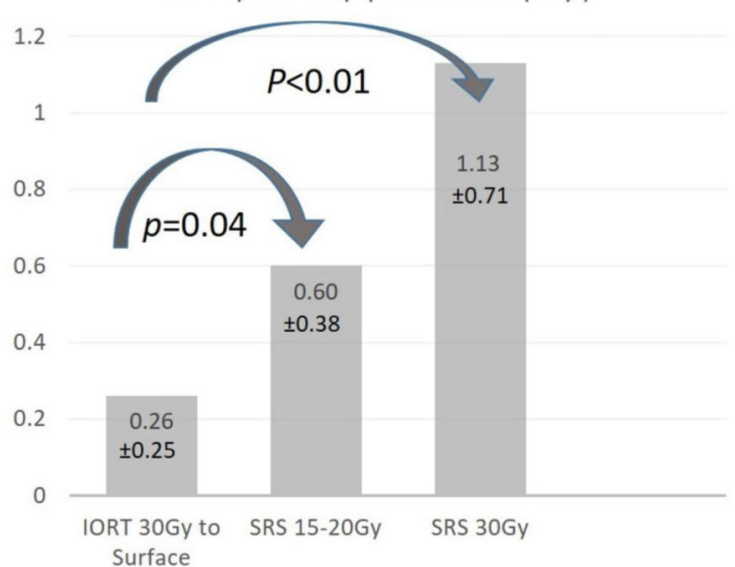

Fig. 2.

Volume of brain receiving $12 \mathrm{~Gy}$ as a function of spherical applicator size and dose prescription. IORT intraoperative radiotherapy, Gy gray, V12 volume of brain receiving 12 Gy 


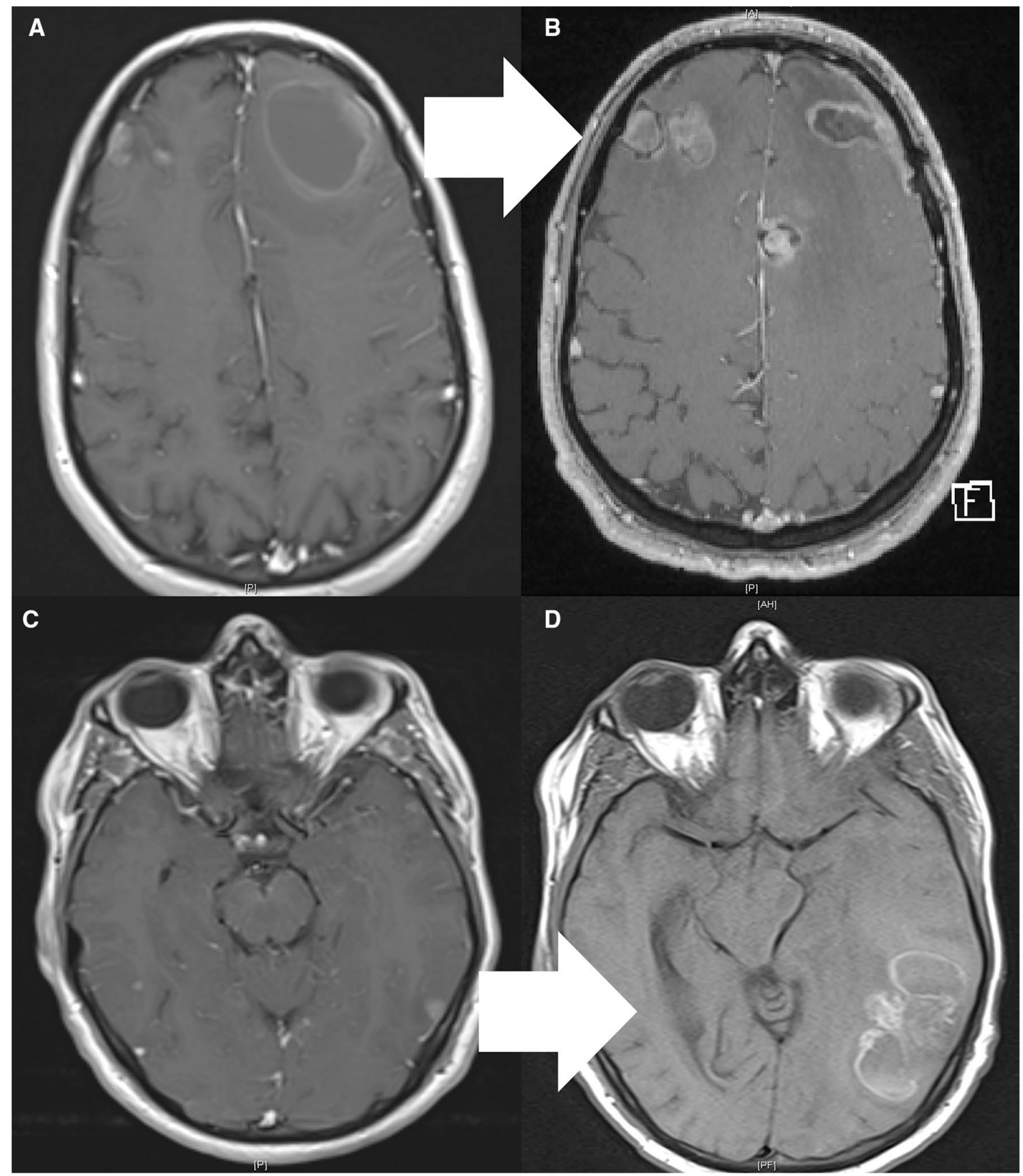

Fig. 3.

Clinical example of the potential favorable outcomes for IORT in reducing radiation injury reactions. a Large left frontal brain metastasis secondary to melanoma treated with surgery and IORT 30 Gy to the applicator surface. b MRI follow-up 9 months after IORT, notice the stable left frontal resection cavity compared to adjacent smaller metastases which were treated with SRS and WBRT with interval associated hemorrhage and radiation injury reaction. Finally compare the outcomes for the left frontal resection cavity from panels a, b to the outcome of a left temporoparietal metastases in panels $\mathbf{c}, \mathbf{d}$ now with significant posttreatment changes and symptomatic mass effect on the adjacent brainstem 


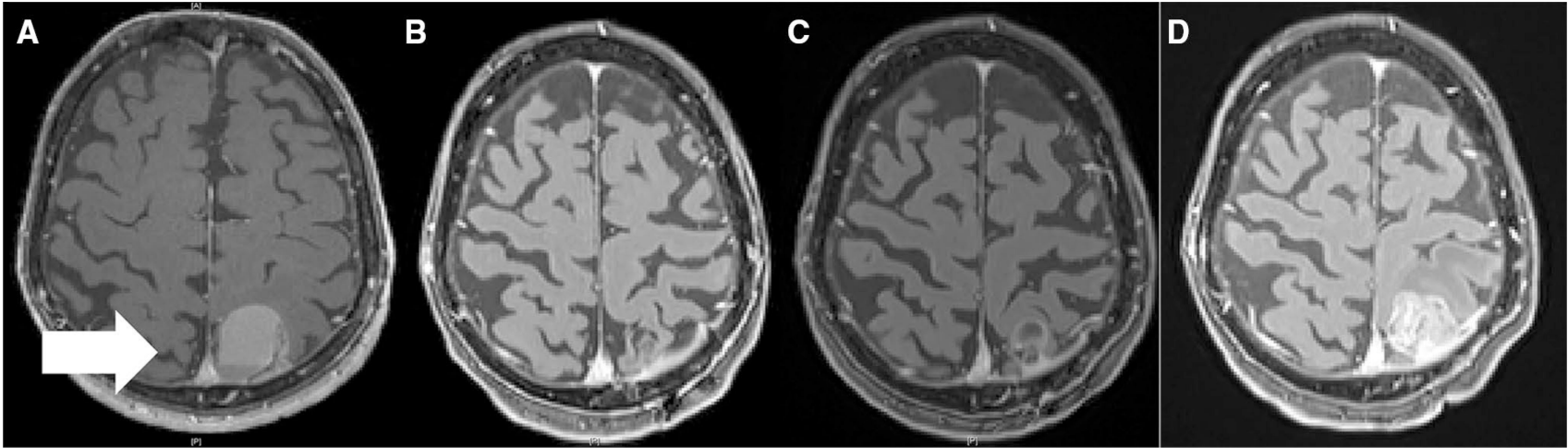

Fig. 4.

Clinical example of local failure after IORT for a dural-base endometrial cancer brain metastasis. a Large left parietal brain metastasis treated with surgery and IORT $30 \mathrm{~Gy}$ to the applicator surface. b Stable resection cavity 2.5 months post-IORT. c Stable to slight increase in enhancement in resection cavity at 5.5 months post-IORT. $\mathbf{d}$ Local recurrence at 8.8 month post-IORT 


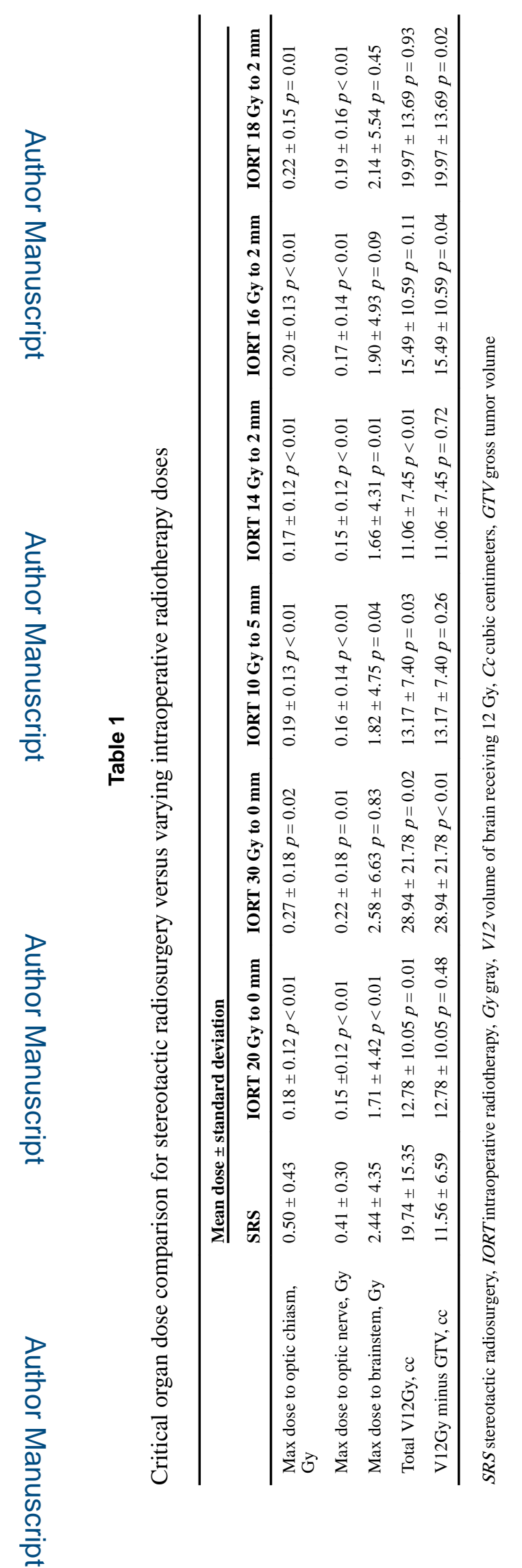

J Neurooncol. Author manuscript; available in PMC 2019 November 01. 